\title{
MAPEO DE LA EVOLUCIÓN DE UNA ENFERMEDAD USANDO SISTEMAS NEURO-DIFUSOS. CASO DE ESTUDIO: ESCLEROSIS MÚLTIPLE
}

\author{
Héctor A. Tabares 0. \\ Instituto Tecnológico Metropolitano ITM. Medellín, Colombia \\ hectortabares@itm.edu.co
}

(Tipo de Artículo: Investigacion. Recibido el 26/03/2012. Aprobado el 08/05/2012)

\begin{abstract}
RESUMEN
El presente artículo propone un modelo para estudiar la evolución de la esclerosis múltiple, enfermedad desmielinizante, neurodegenerativa y crónica del sistema nervioso central. El modelo planteado se basa en la utilización de un sistema neurodifuso como herramienta para describir la progresión de la enfermedad, empleando un caso particular para su validación. Los datos de estudio corresponden a la historia clínica de un paciente con diagnóstico de esclerosis múltiple desde 2003, quien ha padecido cinco episodios críticos. El modelo desarrollado permitió detectar los cambios de la inflación neurológica del paciente.
\end{abstract}

Palabras clave

Esclerosis múltiple, sistema neuro-difuso.

\section{MAPPING OF DISEASE EVOLUTION USING NEURO-FUZZY SYSTEMS. CASE STUDY: MULTIPLE SCLEROSIS}

\section{ABSTRACT}

The aim of this paper is to propose a model for studying multiple sclerosis disease evolution. Multiple sclerosis is a central nervous system, demyelinating, neurodegenerative and chronic disease. The proposed model is based on the use of a neurofuzzy system as a tool for describing the progression of the disease. A specific case is used for the validation of the model. The data used for the study correspond to the medical record of a patient suffering multiple sclerosis since 2003; this patient faced five serious episodes during this time. The model that has been developed allows detecting changes in neurological inflation during treatment.

\section{Keywords}

Multiple sclerosis, neuro-fuzzy system, forecasting.

\section{CARTOGRAPHIE DE L'ÉVOLUTION D'UNE MALADIE EN UTILISANT SYSTĖMES NEURO-FLOUS. ÉTUDE DE CAS : SCLÉROSE MULTIPLE}

\section{RÉSUMÉ}

Cet article propose un modèle pour étudier l'évolution de la sclérose multiple, qui est une maladie démyélinisant, neurodégénérative et chronique du système nerveux central. Le model proposé est basé sur l'utilisation d'un système neuro-flou comme un outil pour décrire la progression de la maladie, en utilisant un cas particulier pour sa validation. Les données de l'étude sont du dossier médical personnel d'un patient diagnostique avec sclérose multiple depuis 2003, qui a éprouvé cinq épisodes critiques. Le model développé permet de détecter les changements de l'inflation neurologique du patient.

\section{Mots-clés}

Sclérose multiple, système neuro-flou. 


\section{INTRODUCCIÓN}

La esclerosis múltiple (EM) es una enfermedad desmielinizante, neuro-degenerativa y crónica del sistema nervioso central. En su génesis actúan mecanismos autoinmunes $y$, a causa de sus efectos, puede tener como consecuencia una movilidad reducida e invalidez en los casos más severos. Después de la epilepsia es la enfermedad neurológica más frecuente entre los adultos jóvenes desde la erradicación de la poliomielitis, y es la causa más frecuente de parálisis en los países occidentales [1]. En [2] se informa que la EM afecta aproximadamente 1 de cada 1000 personas con edades entre 20 y 40 años, más comúnmente en las mujeres.

Con el propósito de estudiar la evolución clínica de la enfermedad, se han empezado a usar nuevas herramientas informáticas inteligentes, tales como las redes neuronales artificiales (RNA) y los sistemas neurodifusos (ND) [3]. Estos últimos combinan las técnicas de las redes neuronales artificiales y las técnicas de inferencia borrosa. La lógica borrosa proporciona un mecanismo de inferencia sobre la incertidumbre y las redes neuronales ofrecen las ventajas computacionales en cuanto al aprendizaje y la generalización. Un detallado estudio sobre los sistemas ND está más allá del ámbito de esta sección. En [4] se ofrece un excelente estudio con referencias específicas.

Un sistema neuronal artificial fue aplicado en [5] para detectar células que producen cáncer de pulmón. A partir de este estudio surgieron otros trabajos en reconocimiento de imágenes en radiología, dermatología, patología, endoscopia [6], análisis de rayos $X$ aplicados en exámenes de mamografía y radiografías de pecho [7].

Dentro de la literatura médica, existen numerosos estudios que emplean modelos ND. En [8], realizaron una clasificación morfológica de imágenes obtenidas con exámenes de resonancia magnética. Consiste en procesar las imágenes usando una RNA para aumentar su tasa de reconocimiento. Como resultado final, se presenta una RNA capaz de distinguir los diferentes contornos de plaquetas producidas por la EM.

Otros estudios evaluaron la aplicación de sistemas ND para el diagnóstico de infarto del miocardio [9, 10], artritis celular [11, 12], tipos de cáncer [13], pronosticar la duración de pacientes en la unidad de cuidados intensivos [14], evolución de enfermedades después de realizarse una operación de trasplantes $[15,16]$. Otros estudios que usan RNA están relacionados con enfermedades cardio-pulmonares y grado de sobrevivencia después del trauma $[17,18]$, interpretación de pruebas de diagnóstico relacionadas con el páncreas [19, 20] y tiroideos [21, 22].

Es por eso como en el Instituto Tecnológico Metropolitano (ITM) se ha venido trabajando en el desarrollo de modelos computacionales [23, 24] que esperan realimentarse con resultados de estudios experimentales y clínicos, para llegar a convertirlos en una herramienta de apoyo para el diagnóstico de una enfermedad. En este caso, se propone un nuevo modelo computacional usando un sistema ND.

En este trabajo se presenta un sistema ND del tipo ANFIS (Adaptative Neuro Fuzzy Inference Systems) para estudiar la evolución de un paciente con padecimiento de EM. La información del paciente fue tomada de su historia clínica y el patrón de evaluación fue escalado según la gravedad de las recaídas. El sistema ND ANFIS fue entrenado y posteriormente validado. El modelo logró estimar la progresión de la enfermedad con un error menor de 0,01 considerado como una buena medida. La creación de este sistema ND le ofrece al médico una herramienta de fácil observación de las lesiones presentadas y de los cambios neurológicos del paciente ante los fármacos aplicados.

Como una forma de contribuir a la confiabilidad del diagnóstico y eficiencia del tratamiento, se propone en este artículo mapear la serie histórica de registros de recaídas usando un sistema ND. Su utilización ayuda al médico a evaluar, de manera más ágil, la variación de la enfermedad y su impacto en el mediano y largo plazo.

\section{DESARROLLO}

\subsection{Patrón cualitativo}

Respetando la confidencialidad de la información médica del paciente y el secreto profesional dentro del marco ético y legal vigente, así como a la custodia de los registros de resonancia magnética y a no ser sujeto de una investigación experimental sin su plena información y libre consentimiento, se presenta a continuación el caso de un paciente anónimo con diagnóstico de EM desde el año 2003, quien ha presentado cinco recaídas entre enero de 2003 hasta marzo de 2010. El paciente recibió dentro de su tratamiento la aplicación de ampollas de Betaferón. La evaluación del tratamiento fue del tipo cualitativo a través de criterios clínicos y seguimiento visual de las lesiones activas mediante exámenes de resonancia magnética cuyos síntomas y observaciones médicas pueden observarse en la Tabla 1.

Tabla 1. Historia clínica, paciente con diagnóstico de EM

\begin{tabular}{|c|c|}
\hline Examen RM & $\therefore 1$, Junio de 2003 . \\
\hline Tipo: Column & cervical \\
\hline Síntomas: & $\begin{array}{l}\text { Dolor en la región cervical irradiado a miembros } \\
\text { superiores y espalda }\end{array}$ \\
\hline Conclusión: & $\begin{array}{l}\text { Se halla mielitis trasversa a la altura de C2-C3 y } \\
\text { C3-C4 }\end{array}$ \\
\hline Examen RM & o: 2, Enero de 2006 \\
\hline Tipo: Cerebra & Columna cervical \\
\hline Síntomas: $\mathrm{Ar}$ & tesia miembros inferiores \\
\hline $\begin{array}{l}\text { Conclusión: } \\
\text { actividad }\end{array}$ & oceso desmielinizante tipo EM con signo de \\
\hline Examen RM & : 3 , Septiembre de 2007 \\
\hline Tipo: Cerebra & Columna cervical \\
\hline Síntomas: $\mathrm{Pa}$ & stesias miembros inferiores \\
\hline $\begin{array}{l}\text { Conclusión: } \\
\text { C3. EM estak }\end{array}$ & $\begin{array}{l}\text { ica desmielinizante lateral izquierda a la altura de } \\
\text { con respecto a control previo de enero de } 2006\end{array}$ \\
\hline
\end{tabular}


Examen RM Nro: 4, Octubre de 2008

Tipo: Cerebral

Síntomas: Parestesias miembros inferiores, Fasciculaciones

Conclusión: Alteraciones se encuentran estables con respecto a la RM3

Examen RM Nro: 4, Octubre de 2010

Tipo: Cerebral

Síntomas: Parestesias miembros inferiores, Fasciculaciones

Conclusión: Paciente con DX EM sin cambios con respecto al estudio de 2008. No hay lesión de reciente aparición y las alteraciones conocidas permanecen estables en el intervalo de tiempo. Evolución satisfactoria

\subsection{Patrón cuantitativo}

Para obtener el patrón de evaluación cuantitativa del paciente, fue necesario escalar de 0 a 100 la severidad de las lesiones provocadas por las recaídas $(0 \mathrm{sin}$ afectación y 100 máximo grado de afectación) cuya información fue tomada de la historia clínica y de una entrevista con el paciente para evaluar el grado de afectación de la enfermedad.

\subsection{Sistema Neurodifuso}

El sistema ND ANFIS propuesto para modelar la evolución de la EM presenta como variable de entrada el tiempo y de salida el grado de progresión de la enfermedad. Se realizaron 23 lecturas de la evolución de la enfermedad, 14 de ellas se utilizaron para realizar el entrenamiento del sistema ND, corresponden a las evaluaciones en los episodios críticos y las 9 restantes (consideradas como episodios no críticos) fueron usadas para la validación. Para las pruebas del sistema ND fue utilizado el ToolBox NeuroFuzzy de MATLAB®. La topología del sistema ND ANFIS seleccionado se puede observar en la Figura 1. Consistió en una neurona de entrada, una neurona de salida, con 5 funciones de membresía (MFs), con funciones de activación Trimf para la entrada de la red y lineal para la salida.

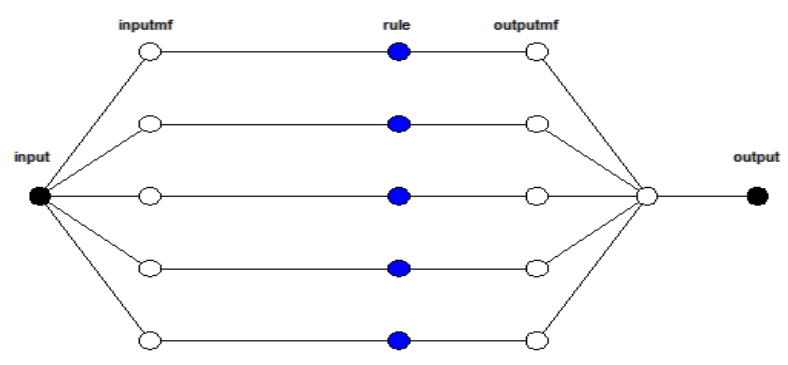

Fig. 1: Topología del sistema ND tipo ANFIS

Los parámetros de los nodos de la capa inputmf son ajustables. Su entrada corresponde a la variable input y la salida del nodo es el grado de pertenencia de dicha entrada. Los nodos de la capa rule calcula el grado de activación de la regla asociada a dicho nodo. La capa outputmf calcula la salida y la última capa combina todas las salidas en un único nodo.

Para entrenar el sistema, se configuró la red para usar el método de optimización híbrido Neuro-fuzzy, error final en la aproximación de 0,01, con 500 iteraciones. Fue empleado el algoritmo de entrenamiento de primer orden gradiente descendente para optimizar los constantes que caracterizan las funciones de pertenencia y el algoritmo de mínimos cuadrados para determinar los parámetros lineales de la salida del modelo de inferencia. Básicamente lo que se hace es generar, a partir de una entrada o salida especifica un sistema de inferencia donde los parámetros de membresía son ajustados usando el algoritmo de retropropagación (red neuronal artificial). Esto provoca que el sistema difuso aprenda de los datos de entrada o salida proporcionados.

Con el propósito de evitar que la red, durante la etapa de entrenamiento, se sobreajuste a los datos, se utilizó el método de detección temprana (early stopping) que emplea dos conjuntos: datos para el entrenamiento y datos para la validación.

\section{RESULTADOS Y DISCUSION}

\subsection{Patrón cuantitativo}

La Tabla 2 presenta el registro histórico de recaídas del paciente, organizado de forma cuantitativa, que incluye: fecha de los episodios, duración y grado de afectación.

Tabla 2. Registro clínico cuantitativo

Fecha: Junio / 2003.

Grado afectación: $100 \%$, AMlyS

Duración: 15 meses

\begin{tabular}{l|l} 
Descripción: & Al finalizar el episodio, el paciente se recuperó
\end{tabular}

Fecha: 2, Enero de 2006

Grado afectación: $100 \%$ AMI

Duración: 12 meses

Descripción: Al finalizar el episodio, el paciente se recuperó en un $85 \%$.

Fecha: Septiembre de 2007

Grado afectación: $50 \%$ AMI

Duración: 8 mese

Descripción: ECP. Sin progresión.

Fecha: Octubre de 2008

Grado afectación: $50 \%$ AMI

Duración: 7 meses

Descripción: ECP. Sin progresión.

Fecha: Marzo de 2010

Grado afectación: 50\% AMI

Duración: 5 meses

Descripción: ECP. Sin progresión.

La Figura 2 representa la progresión cuantitativa de la enfermedad.

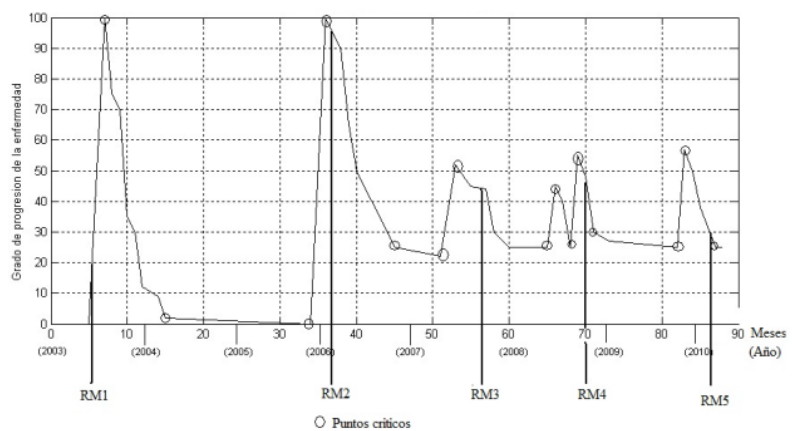

Fig. 2: Progresión de la enfermedad esclerosis multiple

\subsection{Sistema neurodifuso tipo ANFIS}

La Figura 3 muestra la curva de la serie histórica del paciente mapeada por el sistema ND ANFIS. Los datos en círculo son los utilizados para entrenar la red y los 
que se presentan en rectángulo, para la validación del entrenamiento.

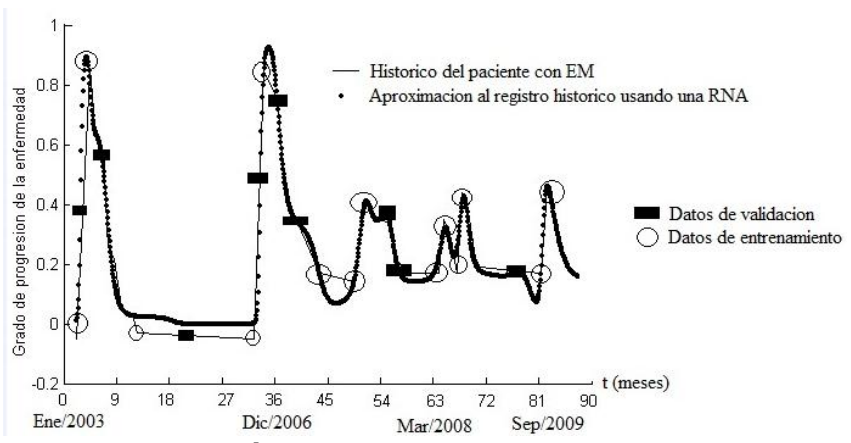

Fig. 3: Evolución de la enfermedad de EM obtenida a partir de la RNA entrenada

El sistema ND mapeó la serie de recaídas logrando una aproximación a la serie histórica en 500 iteraciones, con un error cuadrático medio de $0,00096863 / 0,01$, lo cual en la literatura es considerado una buena medida. Ohno [3] realizó un trabajo similar. La RNA creada por Ohno contó con 24 neuronas en la capa de entrada, cada una con un ejemplo de entrenamiento (lo que presupone una complejidad de función relativamente sencilla), y cuatro neuronas de salida. Con este método no se puede realizar futuras predicciones por cuanto solo se utilizó un ejemplo para el entrenamiento de la red.

La red propuesta en este artículo logró mapear la historia clínica de un paciente con EM, utilizando una variable de entrada y una de salida cada una agrupando un total de 23 registros. En este caso la complejidad de la función a ser aproximada es mucho mayor, facilitando así mismo realizar futuras estimaciones.

\section{CONCLUSIONES}

Este trabajo permitió desarrollar un modelo basado en sistemas ND tipo ANFIS para estudiar la evolución de un paciente con padecimiento de EM. El modelo logró estimar la progresión de la enfermedad con un error de 0,00096863 en la aproximación a la serie histórica de recaídas, lo cual es considerado como una buena medida. El sistema ND le ofrece al médico una herramienta de fácil observación de los cambios neurológicos del paciente ante los fármacos aplicados y lesiones presentadas. La efectividad para modelar la progresión de la EM usando un sistema ND dependerá de la correcta medición de la frecuencia de recaídas y del grado de progresión de la enfermedad.

El método expuesto puede ser aplicado de manera generalizada para estudiar la evolución de cualquier enfermedad, cuando se dispone de los registros históricos con el cual mapear su progresión.

\section{AGRADECIMIENTOS}

El autor reconoce la ayuda del médico José Fernando Zapata del Instituto Neurológico de Antioquia, de la profesora María Elena Moncada, investigadora del ITM y de Leonel Velásquez, director Ingeniería de Sistemas del ITM.

\section{REFERENCIAS}

[1] A. Gutiérrez et al. "Esclerosis Múltiple". R. Zurek (Ed.), Consensos en Neurología: guías de práctica clínica. Exlibris Editores, Vol. I, pp.1-13, 2001.

[2] American Academic of Neurology. "Isocoric Pupil Dysfunction". Continuum. Lifelong learning in neurology, Vol.10, No. 6, pp. 213-217, 2004.

[3] L. Ohno. "Modular neural networks for medical prognosis: quantifying the benefits of combining neural networks for survival prediction". Connection Science: Journal of Neural Computing, Artificial Intelligence and Cognitive Research, Vol. 9, No. 1, pp.71-86, 1997.

[4] B. Martin del Brio \& A. Sanz. "Redes Neuronales y Sistemas Difusos". Alfa-Omega, 2002.

[5] Y. Chiou \& Y. Lure. "Hybrid lung module detection (HLND) system". Cancer Letters, Vol. 77, No. 2-3, pp. 119-126, 1994.

[6] C. Sthepan et al. "Three new serum markers for prostate cancer detection within a percent free PSAbased artificial neural network". Prostate, Vol. 66, No. 6. pp. 651-657, 2006.

[7] Y. Wang \& J. Shiun. "Artificial Neural Network to Predict Skeletal Metastasis in Patients with Prostate Cancer". Medical Systems, Vol. 33, No. 2, pp. 91-100, 2009.

[8] A. Celona; G. Grasso \& L. Puccio. "Artificial Neural Network (ANN) Morphological Classification by Euclidean Distance Histograms for Prognostic Evaluation of Magnetic Resonance Imaging in Multiple Sclerosis". Proceedings SIMAI Congress, Vol. 3, pp. 283-292, 2009.

[9] F. Buarque. "Multiple Sclerosis Plaque: Computer Model and Simulations". Internal Report, Imperial College of Science Technology and Medicine, 2000.

[10] D. Bizios; A. Heij \& B. Bengtsson. "Trained artificial neural network for glaucoma diagnosis using visual field data: A comparison with conventional algorithms". Journal of Glaucoma, Vol. 16, No. 1, pp. 20-28, 2007.

[11] M. Astion et al. "Application of neural networks to the classification of giant cell arteritis". Arthritis and Rheumatism, Vol. 37, No. 5, pp.760-70, 1994.

[12] K. Borges; R. Moura \& A. Steiner. "Diagnosis of Headache using Artificial Neural Networks". International Journal of Computer Science and Network Security, Vol. 10, No.7, pp. 172-178, 2010.

[13] V. Bourdes et al. "Breast cancer predictions by neural networks analysis: a comparison with logistic regression". Proceedings 29th Annual International Conference Medicine and Biology Society, pp. 54245427, 2007.

[14] D. Cook. "Artificial Neural Networks to Predict Mortality in Critical Care Patients: An Application of Supervised Machine Learning". Australasian Anesthesia, Vol. 122, No 2, pp. 173-179, 2005.

[15] H. Doyle et al. "Predicting outcomes after liver transplantation. A connectionist approach". Annals of Surgery, Vol. 219, No. 4, pp. 408-415, 1994.

[16] D. Gil et al. "Application of artificial neural networks in the diagnosis of urological dysfunctions". Expert Systems with Applications, Vol. 36, No. 3, pp. 57545760, 2009.

[17] M. Ebell. "Artificial neural networks for predicting failure to survive following in-hospital cardiopulmonary resuscitation". Journal of Family Practice, Vol. 36, No. 3, pp. 297-303, 1993.

[18] R. Harrison \& L. Kennedy. "Artificial Neural Network Models for Prediction of Acute Coronary Syndromes Using Clinical Data from the Time of Presentation". Annals of Emergency Medicine, Vol. 46, No. 5, pp. 431440, 2005. 
[19] C. Kazmierczak; G. Catrou \& F. Van Lente. "Diagnostic accuracy of pancreatic enzymes evaluated by use of multivariate data analysis". Clinical Chemistry, Vol. 39, No. 9, pp. 1960-1965, 1993.

[20] A. Bartosch; B. Andersson \& J. Nilsson. "Artificial neural networks in pancreatic disease". British Journal of Surgery, Vol. 95, No. 7, pp. 817-826, 2008.

[21] P. Sharpe et al. "Artificial neural networks in diagnosis of thyroid function from in vitro laboratory tests". Clinical Chemistry, Vol. 39, No. 11, pp. 2248-2253, 1993.

[22] A. Gannous \& R. Elhaddad. "Improving an Artificial Neural Network Model to Predict Thyroid Bending Protein Diagnosis Using Preprocessing Techniques". World Academy of Science, Engineering and Technology, Vol. 74, pp. 126-130, 2011.
[23] M. Moncada \& H. Cadavid. "Estimación de variables eléctricas en un muslo 3D con fractura de diáfisis femoral estimulado magnéticamente". Revista Facultad de Ingeniería Universidad de Antioquia, No. 42, pp. 120-131, 2007.

[24] M. Moncada et al. "Desarrollo experimental y computacional para estimar variables eléctricas inducidas en muestras de fémur bovino estimuladas por campos magnéticos de baja frecuencia". Revista Cubana de Investigaciones Biomédicas, Vol. 27, No. 2 , pp. 1-9, 2008 